\title{
Role of Intranasal Steroid in the Prevention of Recurrent Nasal Symptoms after Adenoidectomy
}

\author{
Tamer S Sobhy
}

\begin{abstract}
Nasal obstruction is one of the main symptoms of adenoid hypertrophy, as they are also presented with chronic rhinorrhea, snoring, hyponasal speech and obstructive sleep disorder. Adenoidectomy can reduce both nasal obstructions and upper respiratory infections. Some patients display clinically significant persistent nasal symptoms even after surgery. Symptoms, such as nasal obstruction or recurrent upper respiratory infections, persist in 19 to $26 \%$ of patients. Factors influencing the outcome of intranasal steroids therapy have not been identified. The treatment with nasal steroids may obtain successful results in children to avoid surgery for adenoid recurrence.
\end{abstract}

Keywords: Intranasal steroids, Prevention of recurrent adenoid, Adenoidectomy.

How to cite this article: Sobhy TS. Role of Intranasal Steroid in the Prevention of Recurrent Nasal Symptoms after Adenoidectomy. Clin Rhinol An Int J 2013;6(2):80-83.

Source of support: The work was approved by the institutional review board of the faculty of medicine at Ain Shams University.

Conflict of interest: None declared

\section{INTRODUCTION}

Nasal obstruction is one of the main symptoms of adenoid hypertrophy, they are also presented with chronic rhinorrhea, snoring, hyponasal speech and obstructive sleep disorder. ${ }^{1}$ Adenoidectomy can reduce both nasal obstructions and upper respiratory infections. However, some patients display clinically significant persistent nasal symptoms even after surgery. Symptoms, such as nasal obstruction or recurrent upper respiratory infections persist in 19 to $26 \%$ of patients. ${ }^{2}$ Adenoidectomy remains a commonly performed procedure although produces short-term benefits. ${ }^{3}$ There are two difficulties that have been described to prevent complete adenoidal removal. Lymphoid tissue in the pharyngeal recess is noted by almost all authors as difficult to remove. ${ }^{4}$ The other difficulty, adenoidal tissue bulging into the posterior choanae, which was addressed by Pearl and Manoukian; ${ }^{5}$ they found choanal adenoids in $9 \%$ of their study group.

Although there are few nonsurgical alternative treatment options, these may be considered in less serious cases. Accordingly, studies about intranasal steroid applications under various protocols have been presented in the literature but none of these studies addressed the efficacy of intranasal steroids to prevent recurrence of adenoid after adenoidectomy.

\section{PATIENTS AND METHODS}

This study was a prospective randomized controlled parallel clinical study. As the study had no connection with any of the manufacturers of the drugs or the pharmaceutical industry at all, it was not possible to obtain a placebo, and therefore the study could not be double-blinded. Simple randomization was done with every other patient consecutively.

The study was approved by the institutional review board of the Otorhinolaryngology Department, Faculty of Medicine, at Ain Shams University in Cairo. Children presented to ENT outpatient clinic at Ain Shams University hospitals during the period from April 2009 to June 2011, diagnosed as adenoid hypertrophy were included in the study. The study included two groups, each included 50 child. Written informed consents from the parents were taken, about the participation of their children in the study.

The diagnosis was based on the symptoms (nasal obstruction, nasal discharge, and/or snoring, and lateral radiographs (enlarged convex bulge in the roof of the nasopharynx compressing the nasopharyngeal airway). Exclusion criteria included the use of intranasal or systemic steroids within the last 1 year, use of any intranasal medication within the previous 2 weeks of entering the study, acute URTI within 2 weeks of entering the study, history of epistaxis, immunodeficiency disorders or hypersensitivity to the mometasone furoate. Also, children were excluded from the study if there is a history of craniofacial neuromuscular or genetic disorder.

Assessment of each child upon entering the study included the following: history and physical examination, parental questionnaire, lateral nasopharyngeal radiograph. All patients under the study had complete head and neck evaluation, including flexible fiberoptic nasal endoscopy (according to the compliance of the child). Due to the difficulty of the use of the flexible endoscopy for all patients, it was not feasible to be used in the assessment of the nasopharynx pre- or postoperatively.

The patients were all assessed before and after adenoidectomy as regard the nasal obstruction, discharge, snoring. These symptoms were all graded as grade 1: mild, grade 2: moderate and grade 3: severe.

The lateral view X-ray of the nasopharynx, the size of the adenoids was graded according to the palatal airway measured from the most convex point of the adenoid tissue to the soft palate, the narrowest distance between the nasopharyngeal soft tissue and the soft palate was taken. Grade 1: $>6 \mathrm{~mm}$, grade 2: 4 to $6 \mathrm{~mm}$, grade $3: 0$ to $3 \mathrm{~mm}$. The lateral radiographs are done before surgery and postoperative after 6 months and after 12 months. 
The parents were asked (using the questionnaire) about their overall satisfaction of the nasal condition of the child after adenoidectomy. They were also asked about the number of upper respiratory tract infections in the previous years and postoperatively.

Adenoidectomy was done using the classic method using the adenoid curette. After adenoidectomy, children were then simply randomized into two groups, group I which included ( 50 children) with postoperative intranasal steroids, and group II (50 children) who had postoperative intranasal saline spray. Patients in group I received 12 weeks course of single intranasal spray administration in each nostril with mometasone furoate (40 mcg/day). After this course, all patients in group I were reassessed to evaluate the efficacy of treatment. All patients or parents were asked to report the degree of the symptom after 2 weeks, 6 months and after 1 year postoperative with the questionnaire that is fulfilled by the parents. No other medication was allowed during the treatment. Patients who used systemic steroids for any other reason were excluded from the study. Patients in group II received intranasal saline nasal spray for the same period (12 weeks) and assessment was done in the same way as group I. All patients were followed up after adenoidectomy after 2 weeks, 6 months and 1 year. Lateral radiographs were done after 6 months and after 1 year in the postoperative period.

\section{Statistical Analysis}

The collected data was revised, coded, tabulated and introduced to a PC using statistical package for social science (SPSS 15.0.1 for windows; SPSS Inc, Chicago, IL, 2001).

\section{RESULTS}

In group I patients with intranasal steroids, there were 48 patients (two patients were excluded from the study with age range from 3 to 13 years (mean age: 7.42 years), there were 27 males $(56.3 \%)$ and 21 females $(43.8 \%)$. While in group II with intranasal saline, included 46 patients (four patients were excluded) with age range from 3 to 13 years (mean age: 5.89 years), this group included 21 males (45.7\%) and 25 females (54.3\%). Demographic data of the patients in both groups are shown in Table 1.

\begin{tabular}{|c|c|c|c|c|c|}
\hline & & \multicolumn{2}{|c|}{$\begin{array}{c}\text { Intranasal steroid } \\
\text { group }\end{array}$} & \multicolumn{2}{|c|}{$\begin{array}{c}\text { Intranasal saline } \\
\text { group }\end{array}$} \\
\hline & & $N$ & $\%$ & $N$ & $\%$ \\
\hline \multirow[t]{2}{*}{ Sex } & Male & 27 & $56.3 \%$ & 21 & $45.7 \%$ \\
\hline & Female & 21 & $43.8 \%$ & 25 & $54.3 \%$ \\
\hline Age & Mean \pm SD & 7.42 & 2.86 & 5.89 & 2.72 \\
\hline
\end{tabular}

As regard with nasal obstruction, highly significant difference were observed toward group I with intranasal steroids, when compared with group II with intranasal saline after 6 months $(p=0.001)$ and after 1 year $(p=0.031)$ postoperatively. However, after 2 weeks in the postoperative period, there was no significant difference between both groups.

Regarding nasal discharge, there was a highly significant difference $(p=0.0001)$ between both groups, toward group I with intranasal steroids after 6 months and after 1 year $(p=0.001)$ in the postoperative period. While after 2 weeks in the postoperative period, there was no significant difference $(\mathrm{p}=1.00)$ between both groups.

Regarding snoring, there was a highly significant difference between both groups after 6 months $(p=0.0001)$ and after 1 year $(p=0.001)$. There was no significant difference after 2 weeks $(p=0.363)$ in the postoperative period.

In group I, comparing data after 1 year with the data of the preoperative period there was a highly significant difference elicited in all the three symptoms $(p=0.0001)$. Comparing data after 1 year with the data after 2 weeks in the postoperative period, there was significant difference regarding nasal obstruction only $(\mathrm{p}=0.0001)$ but no difference regarding nasal discharge and snoring. When comparing data after 1 year with the data after 6 months, there was significant difference as regard the three symptoms with $p=0.011$ in nasal obstruction, $p=0.008$ in nasal discharge and $p=0.003$ in snoring. This can be illustrated in Graph 1.

Significant difference $(p=0.003)$ was noted toward group I patients regarding nasopharyngeal radiograph after 1 year in the postoperative period. However, there was no significant difference between both the groups $(p=0.191)$ after 6 months postoperatively.

In group I patients, there was a highly significant difference $(p=0.0001)$ of the nasopharyngeal radiographs when compared the data after 6 months with the data in the preoperative period. A highly significant difference $(p=0.0001)$ could be elucidated when compared the data after 1 year with the data in the preoperative period although failed to find any difference of the radiograph data after 1 year when compared with the data after 6 months which is shown in Graph 2.

In the current study, it was found that the overall satisfaction among parents of group I patients was $85.4 \%$. Overall satisfaction among parents of group II was $76.1 \%$. When comparing the overall satisfaction between both groups, there was no statistically significant difference. Also, there was a highly significant difference of the number of URTI when compared data of the pre- and postoperative 


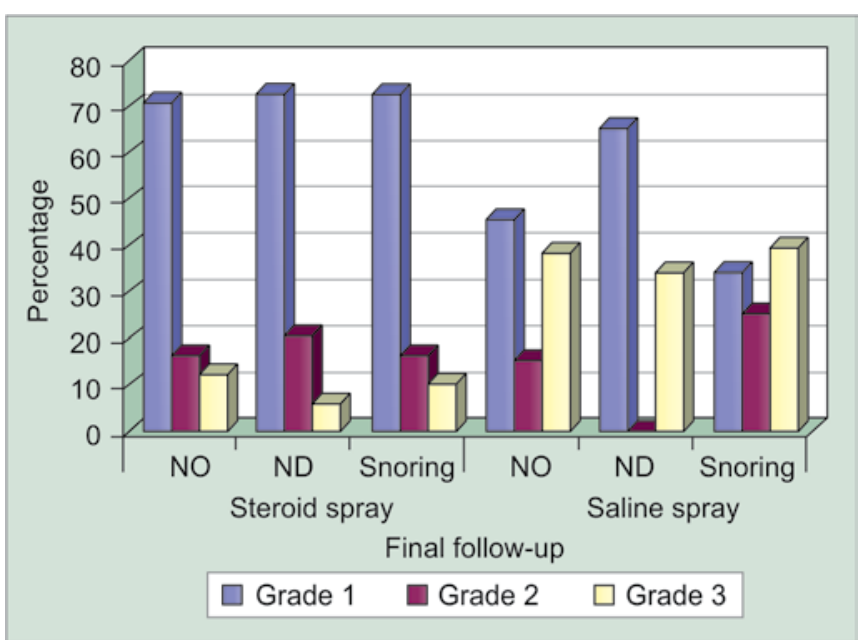

Graph 1: Description of nasal symptom grades among steroid and saline patients at the last follow-up, 1 year postoperative. This graph shows the differences between the nasal symptoms grades between both study groups after 1 year (NO: nasal obstruction, ND: nasal discharge)

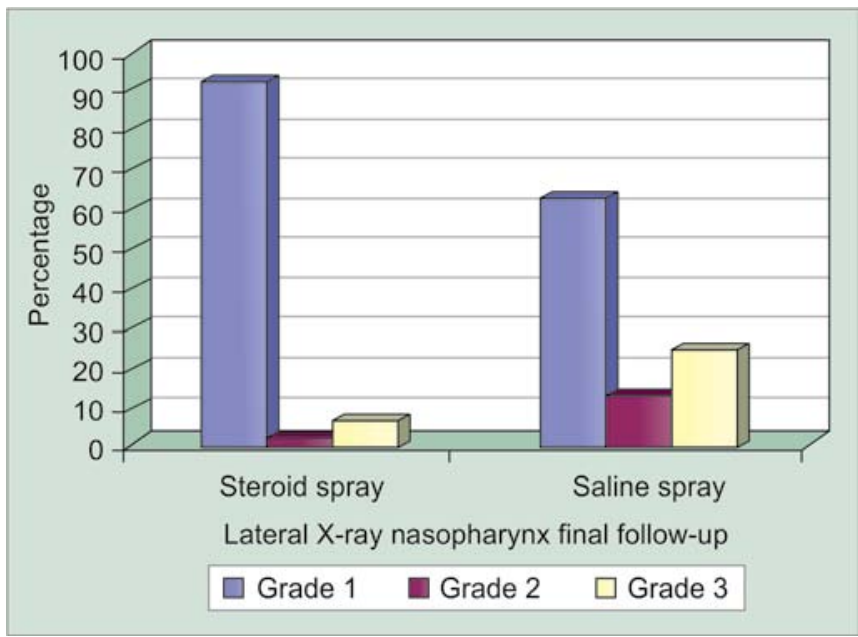

Graph 2: Description of $X$-ray findings among steroid and saline patients at the last follow-up, 1 year postoperative. This graph shows the differences between patients, as regard the adenoid enlargement grade between both study groups after 1 year

periods. The same data was found in group II with a significant difference $(\mathrm{p}=0.0001)$.

There were no intraoperative or postoperative complications in group I. While in group II, postoperatively, three patients developed 2ry bleeding after 7 days.

\section{DISCUSSION}

Revision adenoidectomies are not unheard of. However, a review of the literature, including some prominent textbooks, does not illuminate the issue or its frequency. ${ }^{4}$ Buchinsky et $\mathrm{al}^{4}$ failed to find a new obstructing adenoid pad after adenoidectomy in a large series of children. While on the contrary, Joshua et $\mathrm{al}^{2}$ found a new obstructing adenoid tissue in the clinical practice. They reported infrequent occurrence of adenoid regrowth after adenoidectomy that causes nasal obstruction which accounts for $3 \%$ of patients with persistent postadenoidectomy symptoms.

Successful use of intranasal steroid treatment in children with adenoid hypertrophy was introduced by Demain and Goetz. ${ }^{6}$ Although it is not yet clear by which mechanisms the steroids reduce the nasal airway obstruction. However, there are some theories such as the anti-inflammatory effect of steroids that help to reduce adenoidal and nasopharyngeal inflammation. ${ }^{6}$

The present study showed that the use of intranasal steroids after adenoidectomy was beneficial to relieve nasal obstruction and prevent recurrence of adenoid after adenoidectomy after a follow-up period of 1 year. There is a difference in age across groups (7.4 in group I and 5.89 in group II) which might account for the difference across groups as the Waldeyer's ring involutes with puberty. However, Buchinsky et al, ${ }^{4}$ failed to find any significant difference between children younger than 10 years and those older than 10 years as the proportion between both groups was identical.

In the current study we could not perform endoscopy for all children although it is now the best diagnostic technique for diagnosis of adenoid-related nasal obstruction because it depends on the age and compliance of the child.

The use of postoperative intranasal steroid gives advantage to avoid the second intervention. This is in contrast to Lepcha et al, ${ }^{7}$ who did not find any significant efficacy of intranasal steroids in improving nasal blockage, nasal discharge, or snoring, although a 5-fold reduction in adenoid size that was observed in intranasal steroid group when compared with the placebo group. However, this difference did not reach a statistical significance.

Steroids are generally well-tolerated in children. Studies showed only one case of episodic nasal bleeding. ${ }^{8}$ The effect of intranasal steroids on growth were studied by Allen et al, ${ }^{9}$ in a randomized, double-blind, placebo-controlled study. The growth rate in prepuberty children had used intranasal steroids for 1 year was reported to be equal to the growth rate of the placebo-control group.

The mechanisms by which topical steroids improve nasal airway obstructive symptoms remain unclear. Three main trials succeeded to demonstrate the improvement of nasal obstruction with reduction of adenoid size with the use of intranasal steroids. ${ }^{6,10,11}$

Nonsurgical alternatives for adenoid hypertrophy are limited to treatment of the coexisting upper airway infections. However, it was reported that treatment with intranasal steroids can decrease the size of adenoid hypertrophy, using beclomethasone, ${ }^{6}$ fluticasone, ${ }^{12}$ and mometasone.${ }^{13}$ Among several commercially available steroid nasal sprays, we selected mometasone furoate for this study. This drug had 
been reported previously not to cause any adverse effects on growth and hypothalamic pituitary adrenal axis. Also, the systemic availability of the drug after topical administration is lower than that of other steroids. ${ }^{14}$

Ciprandi and et al, ${ }^{15}$ found that the use of intranasal flunisolide was associated with a significant reduction of adenoid hypertrophy in $72.6 \%$ of the children. On the contrary, isotonic saline solution was associated with a nonsignificant improvement of adenoid hypertrophy as reported in $30.7 \%$ of children. A recent study provided evidence that treatment with nasal steroids could represent for some children an effective means of avoiding adenoidectomy. ${ }^{16}$ The current study also clarified similar results as Ciprandi et al, ${ }^{15}$ as there was significant reduction in the size of the adenoid in lateral radiographs after 1 year with a p-value of 0.003 .

In this study, it was found that the overall satisfaction among parents of group I patients was $85.4 \%$. Similar results were reported by Lesinskas et al, ${ }^{1}$ they showed that $82.7 \%$ of the parents were satisfied with the results of adenoidectomy.

The duration of treatment with intranasal steroids in previous studies varied from 8 to 24 weeks. None of these trials established the optimal duration of treatment in children. The effects are expected after 2 weeks of the initiation of the treatment as described by Criscuoli. ${ }^{16}$

\section{CONCLUSION}

Factors influencing the outcome of intranasal steroids therapy have not been identified. However, this treatment may obtain successful results in children to avoid surgery for adenoid recurrence. The most appropriate drug, the most efficient dose and optimal treatment duration need to be investigated and determined.

\section{ACKNOWLEDGMENTS}

The author is thankful to the entire staff of the Clinic of Otorhinolaryngology at Ain Shams University Hospitals for their help in performing this study. Also, the author thanks Dr Walid Salah, Lecturer of Community Medicine, for his generous statistical help.

\section{REFERENCES}

1. Lesinskas E, Drigotas M. The incidence of adenoidal regrowth after adenoidectomy and its effects on persistent nasal symptoms. Eur Arch Otorhinolaryngol 2009 Apr;266(4): 469-473.

2. Joshua B, Bahar G, Sulkes J, Shpitzer T, Raveh E. Adenoidectomy: long-term follow-up. Otolaryngol Head Neck Surg 2006 Oct;135(4):576-580.

3. Vandenberg SJ, Heatley DG. Efficacy of adenoidectomy in relieving symptoms of chronic sinusitis in children. Arch Otolaryngol Head Neck Surg 1997 Jul;123(7):675-678.
4. Buchinsky FJ, Lowry MA, Isaacson G. Do adenoids regrow after excision? Otolaryngol Head Neck Surg 2000 Nov; 123(5): 576-581.

5. Pearl AJ, Manoukian JJ. Adenoidectomy: indirect visualization of choanal adenoids. J Otolaryngol 1994 Jun;23(3):221-224.

6. Demain JG, Goetz DW. Pediatric adenoidal hypertrophy and nasal airway obstruction: reduction with aqueous nasal beclomethasone. Pediatrics 1995 Mar;95(3):355-364.

7. Lepcha A, Kurien M, Job A, Jeyaseelan L, Thomas K. Chronic adenoid hypertrophy in children-is steroid nasal spray beneficial? Ind J Otolaryngol Head Neck Surg 2002 Oct-Dec;54(4):280-284.

8. Berlucchi M, Valetti L, Parrinello G, Nicolai P. Long-term follow-up of children undergoing topical intranasal steroid therapy for adenoidal hypertrophy. Int J Pediatr Otorhinolaryngol 2008 Aug;72(8):1171-1175.

9. Allen DB, Meltzer EO, Lemanske RF Jr, Philpot EE, Faris MA, Kral KM, Prillaman BA, Rickard KA. No growth suppression in children treated with the maximum recommended dose of fluticasone propionate aqueous nasal spray for one year. Allergy Asthma Proc 2002 Nov-Dec;23(6): 407-413.

10. Berlucchi M, Salsi D, Valetti L, Parrinello G, Nicolai P. The role of mometasone furoate aqueous nasal spray in the treatment of adenoidal hypertrophy in the pediatric age group: preliminary results of a prospective randomized study. Pediatrics 2007 Jun; 119(6):1392-1397.

11. Demirhan H, Aksoy F, Ozturan O, Yildirim YS, Veyseller B. Medical treatment of adenoid hypertrophy with 'fluticasone propionate nasal drops'. Int J Pediatr Otorhinolaryngol 2010 Jul;74(7):773-776.

12. Brouilette RT, Manoukian JJ, Ducharme FM, Oudjhane K, Eark LG, Laden S, Morielli A. Efficacy of fluticasone nasal spray for pediatric obstructive sleep apnea. J Pediatr 2001 Jun;138(6): 838-844.

13. Cengel S, Akyol MU. The role of topical nasal steroids in the treatment of children with otitis media with effusion and or adenoid hypertrophy. Int J Pediatr Otorhinolaryngol 2006 Apr;70:639-645.

14. Jung YG, Kim HY, Jin-Young Min, Dhong HJ, Chung SK. Role of intranasal topical steroid in pediatric sleep disordered breathing and influence of allergy, sinusitis and obesity on treatement outcome. Clin Experiment Otorhinolaryngol 2011 Mar;4(1):27-32.

15. Ciprandi G, Varrichio A, Capasso M, Varricchio AM, De Lucia A, Ascione E, Avvisti F, Capristo C, Marseglia GL, Barillari U. Intranasal flunisolide treatment in children with adenoidal hypertrophy. Int J Immunopathol Pharmacol 2007 Oct-Dec;20(4):837-840.

16. Criscuoli G, D'Amora S, Ripa G, Cinquegrana G, Mansi N, Impagliazzo N, Pisacane A. Frequency of surgery among children who have adenotonsillar hypertrophy and improve after treatment with nasal beclomethasone. Pediatrics 2003 Mar;111(3):236-238.

\section{ABOUT THE AUTHOR}

\section{Tamer S Sobhy}

Lecturer, Department of Otorhinolaryngology, Faculty of Medicine Ain Shams University, Cairo, Egypt

Correspondence Address: 15 El-Khalifa El-Maamoun, Heliopolis Cairo, Egypt, Phone: 0020222911674, 0020201007233186, 0020201007046655, e-mail: tamshok2008@hotmail.com 